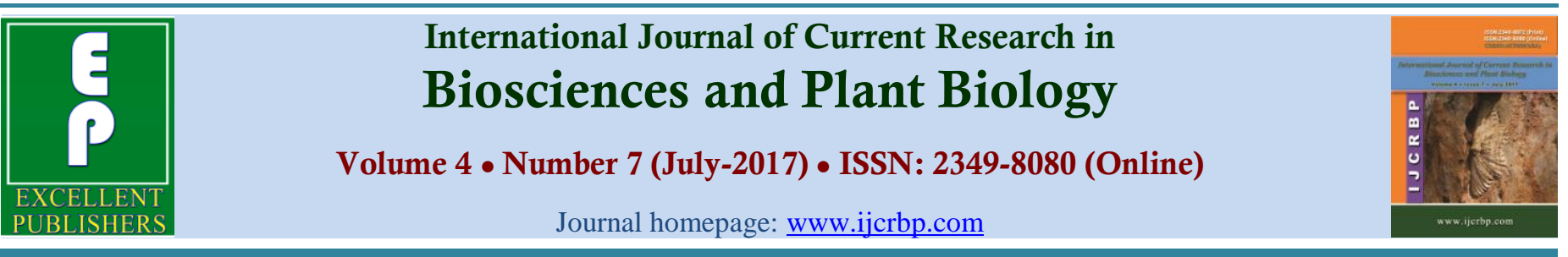

\title{
Effect of Exercise Program and Relaxation Techniques on Fatigue and Physical Performance in Cancer (Haematology) Patients Undergoing Chemotherapy
}

\author{
Rupali Biswas (Sen)*, Maitrayee Bhattacharya and Abisekh Biswas
}

Department of Haematology, N R S Medical College and Hospital, Kolkata, West Bengal-700 014, India

*Corresponding author.

\begin{tabular}{|c|c|}
\hline Abstract & Article Info \\
\hline \multirow{7}{*}{$\begin{array}{l}\text { A total number of } 30 \text { Haematology cancer subjects with } 15 \text { each in control and } \\
\text { experimental group from Day Care Centre of Department of Haematology, N R S Medical } \\
\text { College and Hospital, Kolkata was sampled by convenient sampling method for a period } \\
\text { of } 12 \text { months. Exercise program along with relaxation techniques were provided to } \\
\text { experimental group and no intervention was provided to control group. Fatigue Severity } \\
\text { Scale (FSS) and Physical Performance Test (PPT) were the two outcome measures used. } \\
\text { Exercise program along with the relaxation techniques yielded significant results i.e., } \\
\text { FSS }<0.002 \text { and PPT }<0.002 \text {, which shows that this exercise program along with relaxation } \\
\text { techniques helps in decrease the fatigue level and improvement in physical performance in } \\
\text { cancer (Haematology) undergoing chemotherapy. The current study has shown that } \\
\text { exercise program along with the relaxation technique decrease fatigue and improve } \\
\text { physical performance in cancer patients which proves our hvpothesis }\end{array}$} & $\begin{array}{l}\text { Accepted: } 01 \text { June } 2017 \\
\text { Available Online: 06 July } 2017\end{array}$ \\
\hline & Keywords \\
\hline & Cancer (Haematology) \\
\hline & Exercise program \\
\hline & Fatigue \\
\hline & Physical performance \\
\hline & Relaxation techniques \\
\hline
\end{tabular}

\section{Introduction}

Cancer is the disordered and uncontrolled growth of cells within a specific organ or tissue type. If left untreated, they grow steadily resulting in a mass, tumour or growth. The tumour may be benign or malignant (Cooper 2006). Cancer and its treatment are often associated with adverse physical side-effects including muscular atrophy, decreased muscle strength and reduced aerobic capacity. These side-effects may contribute to the development of cancer-related fatigue. About $70 \%$ of cancer patients report fatigue complaints during chemotherapy and/or radiotherapy. Even years after the treatment, fatigue is still a problem for up to
$30 \%$ of cancer survivors and has a great impact on the patient's quality of life (van Weert et al., 2005).

Worldwide, there were 10.9 million new cases of cancer, 6.7 million deaths and 24.6 million people living with cancer (within three years of diagnosis) in 2002. It is estimated that the number of new diagnoses will increase to 95,000 new cases in 2015, representing an increase of approximately 40\% (Helgeson and Cohen, 2012).

A total national cancer incident causes in 2008 were 178,816 cases in Korea. Stomach cancer $15.7 \%$ ) was most prevalent followed by thyroid cancer (15.1\%) 
colorectal cancer $(12.7 \%)$, lung cancer $(10.5 \%)$, liver cancer $(8.8 \%)$, and breast cancer $(7.1 \%)$.

Haematological malignant occur in 8006 patients which has $4.5 \%$ of all cancers next to breast cancer. It occurred in $4.1 \%$ of all cancers occurred in women. Among all hematologic malignant non-Hodgkin lymphoma (NHL) was most important and it was followed by myeloid leukaemia and multiple myeloma regardless of gender.

Ages between 60 and 69 were most prevalent followed by ages 70 to 79 and 50 to 59.Lymphoidleukomia was most prevalent in ages above 15 years (Dimeo et al., 1999).

American Cancer Society estimated new cases of Leukaemia 35\%, Myeloma-18\%, Lymphoma- $47 \%$ and total cases $-1,71,550$. Cancer is the second leading cause of premature death in India. In India it is estimated that there are approximately $2-2.5$ million cases of cancer at any given point of time with around 7, 00,000 new cases being detected each year. The four most frequent cancers in males in India are blood cancer, mouth or pharynx, oesophagus, stomach and lower respiratory tract. Among Indian woman, cancer of the cervix and breast account nearly 60 percent of all cancers (Norum and Wist, 1996).

Most cancer patients experience a loss of energy and an impairment of physical performance. Some authors estimate that this problem affects up to $70 \%$ of cancer patients during chemo- and radiotherapy or after surgery. Furthermore, up to $30 \%$ of cancer survivors have been reported to experience a loss of energy for years after cessation of treatment. For many patients, fatigue is a severe and activity-limiting symptom. This impairment in physical fitness is a significant contributor to decreased quality of life .In response to fatigue; patients are often advised to rest and to down regulate their level of daily activities. But since inactivity induces muscular catabolism, prolonged rest can actually help perpetuate fatigue (Dimeo et al., 1998).

Occupational therapists make a valuable contribution in alleviating certain symptoms, the intervention being symptom-led rather than disease- or diagnosis-led. Because occupational therapy is concerned with the functional implications of symptoms, the occupational therapist also tries to plan ahead and anticipate functional problems rather than waiting for a crisis to occur. Exercise training has been introduced to improve physical capacity and to reduce fatigue. Few intervention studies have included patients who were undergoing chemotherapy and the evidence is modest (Schmitz et al., 2005).

Relaxation (rhythmic contraction and relaxation of large muscle masses over an extended time) has been shown to improve physical performance in cancer patients. In recent few studies we observed a reduction in the fatigue reported by cancer patients after participation in an aerobic training program designed to improve physical performance. The growing absolute numbers of cancer survivors means there is the potential for economic and occupational impact and effects should fatigue persist even in the minority. There is also the additional increased use of healthcare resources as a secondary problem (Durak et al., 2001).

Therefore the present study aimed to find out whether the exercise program and relaxation techniques in cancer (Haematology) patients undergoing chemotherapy, provides beneficial effects on fatigue and physical performance or not.

\section{Aim and objectives}

To find out the effect of exercise program and relaxation techniques on fatigue and physical performance in cancer (Haematology) patients undergoing chemotherapy.

\section{Hypothesis}

Relaxation technique along with graded exercise program is highly effective in reducing fatigue level and improving physical performance in cancer patients undergoing chemotherapy.

\section{Null hypothesis}

Relaxation technique along with graded exercise program is not effective in reducing fatigue level and improving physical performance in cancer patients undergoing chemotherapy.

\section{Background of the study}

1. Dimeo et al. in the year 2004 conducted a study on "Effect of aerobic exercise and relaxation training on fatigue and physical performance of cancer patients after surgery" and concluded that a structured aerobic training 
programme improves the physical performance of patients recovering from surgery for solid tumours. However, exercise is not better than progressive relaxation training for the treatment of fatigue in this setting.

2. Adamsen et al. (2009), conducted a study on Effect of a multimodal high intensity exercise intervention in cancer patients undergoing chemotherapy: randomised controlled trial" and they concluded that a supervised multimodal exercise intervention including high and low intensity components was feasible and could safely be used in patients with various cancers who were receiving adjuvant chemotherapy or treatment for advanced disease. The intervention reduced fatigue and improved vitality, aerobic capacity, muscular strength, and physical and functional activity, and emotional wellbeing, but not quality of life.

3. Purcell co-workers in the year 2009 conducted a study on "Cancer-related fatigue (CRF) is reported as the most common and distressing symptom experienced by patients with cancer (National Comprehensive Cancer Network)". It may affect previous ability to work and to care for himself or herself and others may lead to increased health care resource use.

4. Beaton et al. in the year 2009 , conducted a study on "Effects of exercise intervention on persons with metastatic cancer" and concluded that exercise may be beneficial for persons with metastatic cancer.

\section{Materials and methods}

Study area: N R S Medical College and Hospital, Kolkata.

Study population: Cancer patients (Haematology) of following categories who were under chemotherapy.

\section{Acute leukemia}

a) Acute Lymphoid Leukemia

b) Acute Myeloid Leukemia

\section{Chronic leukemia}

a) Chronic lymphocytic leukemia

b) Lymphoma and MM

Study period: 12 months.

\section{Sample size:}

- Total no .of subjects were 30 .

- Among them subjects in experimental group were 15 and subjects in control group were 15 .

Study design: Convenient sampling, controlexperimental group design.

\section{Inclusion criteria:}

1. Subjects with carcinoma (haematology) having following criteria:

- Total count $-4,000-11,000 / \mathrm{cmm}$

- $\mathrm{Hb}-8 \mathrm{gm} / \mathrm{dl}$

- Platelet count -over $50,000 / \mathrm{cmm}$

- Endurance level-minimum 50\% (50 ft walk test-25ft in front and $25 \mathrm{ft}$ in back)

2. Age group: $18-55$ years

3. Ability to understand and follow the command as well as cooperative.

4. Hematological cancer patients will be grouped according to the underlying diseases:

5.

\section{Acute leukemia}

a) Acute Lymphoid Leukemia

b) Acute Myeloid Leukemia

2. Chronic leukemia

a) Chronic lymphocytic leukemia

b) Lymphoma and MM

Exclusion criteria: Subjects having

1. Any other associated problems like Cardiac disease.

2. Surgical intervention on other reasons.

3. Any other type of cancer other than hematology.

\section{Outcome measures:}

1. Fatigue Severity Scale (FSS)

2. Physical Performance Test (PPT)

Ethical approval for the study: Ethical Committee of NRS Medical College and Hospital, Kolkata.

\section{Methods of study technique}

Control group: No intervention

Experimental group: 1. Relaxation; 2. Exercise therapy. 


\section{Relaxation}

A. Cognitive Behavioral Therapy (cognitive behavioural approach like meditation, Assertive Training)

B. Somatic methods of relaxation
i. Progressive relaxation training.
ii. Breathing.

\section{Exercise therapy}
A. Active movements
B. Flexibility exercises
C. Resistive exercises

\section{Duration of intervention:}

- Alternate days in a week (Monday, Wednesday and Friday) for 12 weeks.

- Control group: no intervention.

- Experimental group: 30 minutes for relaxation and 30 minutes for exercises.

\section{Flow Chart of Methodology}

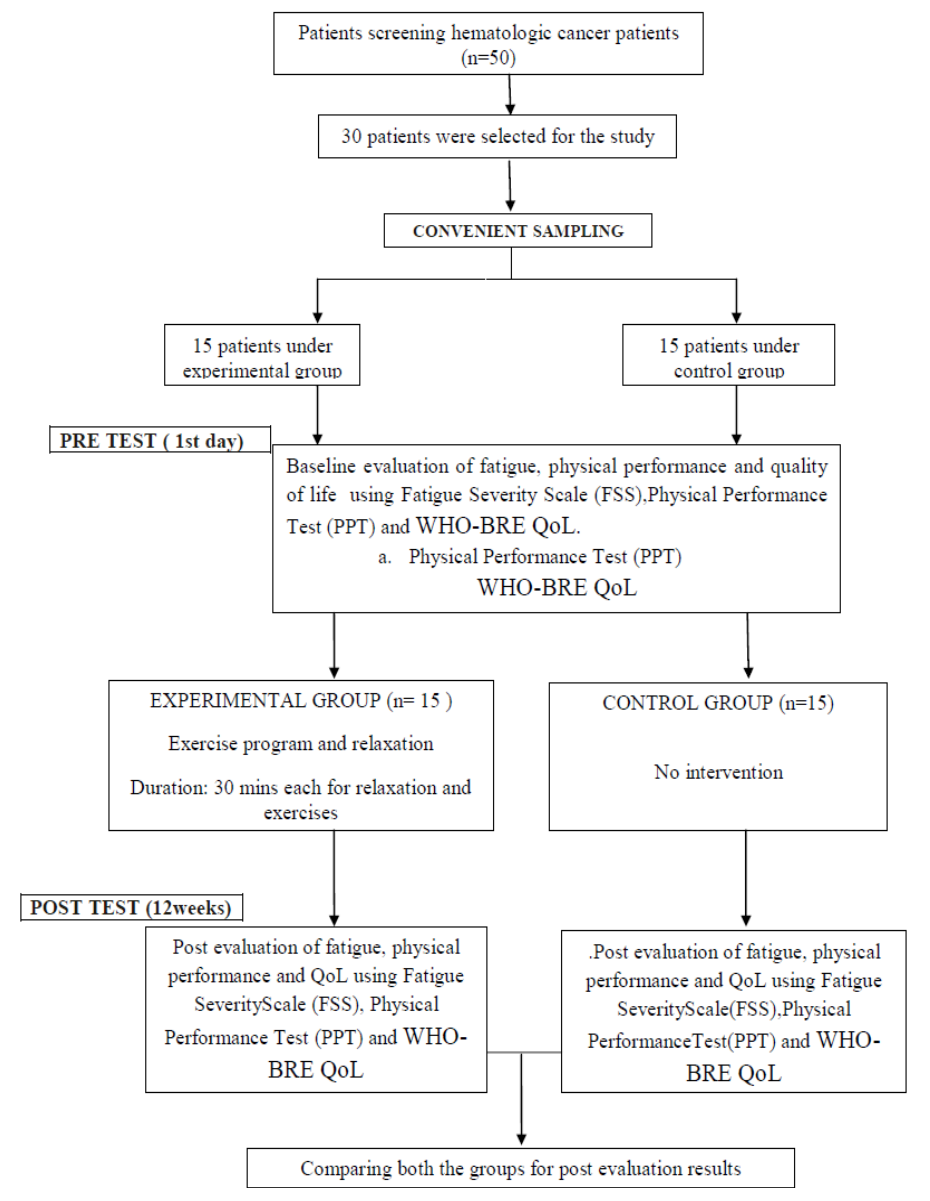

\section{Plan for analysis of data}

Data was analyzed by using Statistical Package for Social Science (SPSS) version 20.

\section{Results and discussion}

A total number of 30 haematology cancer patients were recruited for the study with age range from 18 to 55 years. There were 26 male and 4 female patients in the study. There was no drop out during the study. Data was collected at the 1st day visit and after the completion of study. The results are shown in Tables 1-2 and Figs. 1-2.

The present study examined the effect of exercise program and relaxation techniques on fatigue and physical performance in cancer (Haematology) patients undergoing chemotherapy.

As haematology patients often complain of fatigue and reduced physical performance following chemotherapy, it was hypothesized that relaxation technique along with graded exercise program is highly effective in reducing fatigue level and improving physical performance in cancer patient undergoing chemotherapy and it can supported by the study of Martina E.Schmidt et al (2015) where effects of resistance exercises on fatigue is highly effective in cancer patients undergoing chemotherapy.

The results were significant $(p<0.05)$ for the Fatigue Severity Scale (FSS) and Physical Performance Test (PPT). After analysis of the obtained data, findings support the experimental hypothesis. In this study the fatigue was measured by the Fatigue Severity Scale which was the first outcome measure and there was a significant improvement in the experimental group and this can accord with the study of Carlson et al. (2006) where Individualized exercise program for the treatment of severe fatigue in patients after allergenic hematopoietic stem-cell transplant showed significant improvement.

Also in the second outcome Physical Performance Test used for measuring physical performance showed significant improvement in the experimental group, this can be supported by the study of Adamsen et al (2006) where they observed highly significant improvements in physical functioning $(p<0.001)$ and role functioning $(p<0.001)$ after a multidimensional exercise intervention on physical capacity, well-being and quality of life in cancer patients undergoing chemotherapy. 
Table 1. Within group analysis of Fatigue Severity Scale (FSS).

\begin{tabular}{lllll}
\hline Group & PRE $($ mean \pm SD) & POST $($ mean \pm SD) & Z-value & $p$-value \\
\hline Control & $46.60 \pm 2.694$ & $46.73 \pm 3.615$ & -0.439 & 0.660 \\
Experimental & $36.27 \pm 7.869$ & $51 \pm 2.330$ & -3.192 & 0.002 \\
\hline
\end{tabular}

Table 2. Within group analysis of Physical Performance Test (PPT).

\begin{tabular}{lllll}
\hline Group & PRE $($ mean \pm SD $)$ & POST $($ mean \pm SD) & Z value & $p$-value \\
\hline Control & $11.20 \pm 1.474$ & $11.40 \pm 1.183$ & -1.134 & 0.257 \\
Experimental & $10.47 \pm 1.125$ & $14.13 \pm 3.137$ & -3.068 & 0.002 \\
\hline
\end{tabular}

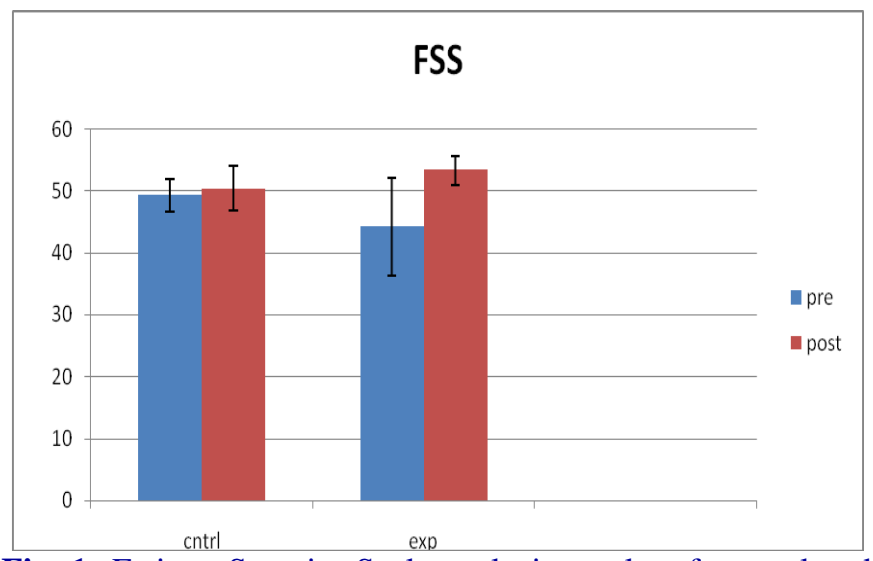

Fig. 1: Fatigue Severity Scale analysis results of control and experimental group (error bars indicate $\pm \mathrm{SD}$ ).

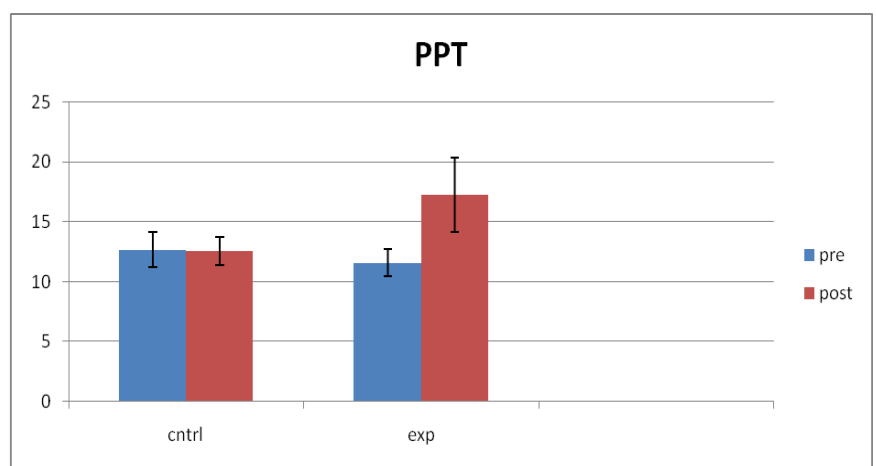

Fig. 2: Physical Performance Test (PPT) results of control and experimental group (error bars indicate $\pm \mathrm{SD}$ ).

Regarding the exercises along with relaxation techniques has significant effect as because after chemo there are many side effects like severe fatigue and reduced strength etc. The comparison of our exercise intervention with a non exercise intervention with other similar conditions enabled us to investigate the pure training effects and this can accord with the study of Ollie et al. (2015).

Strength of this study include a well defined supervised training with minimal dropout rate and these favourable findings hypothesized that relaxation techniques and exercise together improves the fatigue and physical performance in haematological cancer patients undergoing chemotherapy.

\section{Study limitations}

This study included only cancer haematology patients and the results may not applicable to the other cancer patients. Future research should investigate for the other type of cancer patients.

\section{Conclusion}

The current study has shown that exercise program along with the relaxation technique decrease fatigue and improve physical performance in cancer (Haematology) patients undergoing chemotherapy which proves our hypothesis. We also continually should raise the awareness amongst all health professionals to introduce a full scale rehabilitation programme if the correct infrastructure is available.

\section{Conflict of interest statement}

Authors declare that they have no conflict of interest.

\section{Acknowledgement}

We would like to express our sincere thanks and deepest gratitude to Doctors, nurses and other staff of Haematological department of N.R.S Medical College and Hospital Kolkata under West Bengal University of Health Sciences, Kolkata for their guidance help and encouragement. We also thank our family members, friends and well wishers for their continuous support throughout the study, which was a great encouragement to us. We would like to thank Ms. Soumyashree Dash (MOT $2^{\text {nd }}$ year) and Ms. Insana (MOT $2^{\text {nd }}$ Year) for her 
cooperation regarding DTP work. Finally, we thank our clients for thrusting us during the course of the study.

\section{References}

Adamsen, L., Morten, Q., Christina, A., Tom, M., Jørn, H., Dorte, K., et al. 2009. Effect of a multimodal high intensity exercise intervention in cancer patients undergoing chemotherapy: Randomised controlled trial. BMJ. 339, b3410.

Adamsen, L., Quist, M., Midtgaard, J., Andersen, C., Møller, T., Knutsen, L., Tveterås, A., Rorth, M., 2006. The effect of a multidimensional exercise intervention on physical capacity, well-being and quality of life in cancer patients undergoing chemotherapy. Support Care Cancer. 14(2), 116-127.

Beaton, R, Pagdin-Friesen, W, Robertson, C, Vigar, C., Watson, H., Harris, S.R., 2009. Effects of exercise intervention on persons with metastatic cancer: A systematic review. Physiother. Can. 61, 141-153.

Carlson, L.E., Smith, D., Russell, J., Fibich, C., Whittaker, T., 2006. Individualized exercise program for the treatment of severe fatigue in patients after allogeneic hematopoietic stem-cell transplant: a pilot study. Bone Marrow Transplant. 37(10), 945-954.

Cooper, J., 2006. Occupational Therapy in Oncology and Palliative Care. $2^{\text {nd }}$ Edn. Chapter 1. p.1.

Dimeo, F., Rumberger, B.G., Keul, J., 1998. Aerobic exercise as therapy for cancer fatigue. Med. Sci. Sports Exerc. 30(4), 475-478.

Dimeo, F.C., Stieglitz, R.D., Novelli-Fischer, U., Fetscher, S., Keul, J., 1999. Effects of physical activity on the fatigue and psychologic status of cancer patients during chemotherapy. Cancer. 85(10), 2273-2277.

Dimeo, F.C., Thomas, F., Raabe-Menssen, C., Pröpper, F., Mathias, M., 2004. Effect of aerobic exercise and relaxation training on fatigue and physical performance of cancer patients after surgery. A randomised controlled trial. Support. Care Cancer. 12, 774-779.

Durak, E.P., Harris, J., Susan, M., 2001. Ceriale the effects of exercise on quality of life improvements in cancer survivors: the results of a national survey. J. Exercise Physiol. 4(4), 21-28.

Helgeson, V.S., Cohen, S., 1996. Social support and adjustment to cancer: Reconciling descriptive, correlational, and intervention research. Health Psychol. 15, 135-148.

Norum, J., Wist, E., 1996. Psychological distress in survivors of Hodgkin's disease. Support Care Cancer. 4, 191-195.

Ollie, M., Jo, F., Jane, M., 2015. The role of behavioural modification and exercise in the management of cancer-related fatigue to reduce its impact during and after cancer treatment. Acta Oncologica. 54(5), 581-586.

Purcell, A., Fleming, J., Haines, T., Bennett, S., 2009. Cancer-related fatigue: A review and a conceptual framework to guide therapists' understanding. British J. Occupat. Ther. 72(2), 79-86.

Schmidt, M.E., Wiskemann, J., Armbrust, P., Schneeweiss, A., Ulrich, C.M., Steindorf, K., 2015. Effects of resistance exercise on fatigue and quality of life in breast cancer patients undergoing adjuvant chemotherapy: A randomized controlled trial. Int $\mathbf{J}$ Cancer. 137(2):471-480.

Schmitz, K.H., Holtzman, J., Courneya, K.S., Mâsse, L.C., Duval, S., Kane, R., 2005. Controlled physical activity trials in cancer survivors: A systematic review and meta-analysis. Cancer Epidemiol. Biomarkers Prev. 14, 1588-1595.

van Weert, E., Hoekstra-Weebers, J., Grol, B., et al., 2005. A multidimensional cancer rehabilitation program for cancer survivors effectiveness on health-related quality of life. J. Psychosom. Res. 58, 485-496.

\section{How to cite this article:}

Rupali, B., Maitrayee, B., Abisekh, B., 2017. Effect of exercise program and relaxation techniques on fatigue and physical performance in cancer (haematology) patients undergoing chemotherapy. Int. J. Curr. Res. Biosci. Plant Biol. 4(7), 75-80. doi: https://doi.org/10.20546/ijcrbp.2017.407.009 